\title{
Anosmia e disgeusia no paciente com coronavírus: revisão narrativa
}

\author{
Anosmia and dysgeusia in the coronavirus patient: narrative review
}

Anosmia y disgeusia en el paciente con coronavirus: revisión narrativa

Melyssa de Carvalho Cardoso ${ }^{1 *}$, Letícia Silva Guimarães ${ }^{1}$, Isaias Jonatha Melo de Andrade ${ }^{1}$, Arthur Handerson Gomes Silva1, Giovana Carla Souza1, Letícia Araújo Menezes Castro¹, Jonas Campos

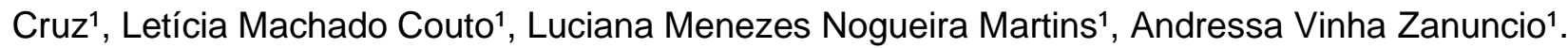

\section{RESUMO}

Objetivo: Estudar e sumarizar estudos que mostram a possibilidade de triagem e isolamento precoce dos casos da COVID-19 (coronavírus disease) pela ocorrência dos distúrbios olfativos e gustativos em pacientes acometidos, de modo a facilitar o acesso a informações sobre o manejo e abordagem da doença. Revisão bibliográfica: A COVID-19, causada pelo SARS-CoV-2 (Severe Acute Respiratory Syndrome Coronavirus 2), surgiu na região leste asiática e tornou-se uma pandemia provocando a morte de milhares de indivíduos. Febre, dispneia, tosse, artralgia, mialgia, cefaleia, rinorreia e diarreia são os sintomas mais prevalentes, além de alterações olfativas e gustativas, que comumente causam a anosmia e disgeusia. Esses distúrbios podem aparecer em conjunto com os demais sintomas ou isoladamente. O período de melhora dos sintomas olfativos, desde o seu início, variou de uma a quatro semanas, com resolução espontânea em sua maioria. Considerações finais: Estudos relacionando a ocorrência dos distúrbios olfativos e gustativos com a COVID19 ainda são escassos, porém tais manifestações têm prevalência considerável, podendo ser indicativas na triagem e isolamento precoce dos casos dessa doença.

Palavras-chave: Coronavírus, Olfato, Paladar.

\begin{abstract}
Objective: To study and summarize studies that show the possibility of screening and early isolation of cases of COVID-19 (coronavirus disease) due to the occurrence of olfactory and gustatory disorders in affected patients, in order to facilitate the access to information on the management and approach to the disease. Bibliographic review: COVID-19, caused by SARS-CoV-2 (Severe Acute Respiratory Syndrome Coronavirus 2), emerged in East Asia and became a pandemic causing the death of thousands of individuals. Fever, dyspnoea, cough, arthralgia, myalgia, headache, rhinorrhea and diarrhea are the most prevalent symptoms, in addition to olfactory and gustatory changes, which commonly cause anosmia and dysgeusia. These disorders can appear together with the other symptoms or isolated. The period of improvement of olfactory symptoms, since its onset, varied from one to four weeks, with spontaneous resolution mostly. Final considerations: Studies relating the occurrence of olfactory and gustatory disorders with COVID-19 are still scarce, however such manifestations have considerable prevalence and maybe indicative in the screening and early isolation of cases of this disease.
\end{abstract}

Keywords: Coronavirus, Smell, Taste.

\footnotetext{
1 Universidade Federal de São João del-Rei Campus CCO (UFSJ CCO), São João del-Rei - MG.

*E-mail:melycardoso@hotmail.com
} 


\section{RESUMEN}

Objetivo: Estudiar y resumir los estudios que muestran La posibilidad de detección y aislamiento temprano de los casos de COVID-19 (enfermedad por coronavirus) debido a la aparición de trastornos olfativos y gustativos en los pacientes afectados, con el fin de facilitar el acceso a la información sobre el manejo y el enfoque de enfermedad. Revisión bibliográfica: COVID-19, causado por el SARS-CoV-2 (coronavirus del síndrome respiratorio agudo severo 2), surgió en el este de Asia y se convirtió en una pandemia que causó la muerte de miles de personas. Fiebre, disnea, tos, artralgia, mialgia, dolor de cabeza, rinorrea y diarrea son los síntomas más frecuentes, además de los cambios olfativos y gustativos, que comúnmente causan anosmia y disgeusia. Estos trastornos pueden aparecer junto con los otros síntomas o de forma aislada. El período de mejora de los síntomas olfativos, desde su inicio, varió de una a cuatro semanas, con resolución espontânea en su mayoría. Consideraciones finales: Los estudios que relacionan la aparición de trastornos olfativos y gustativos con COVID-19 aún son escasos, sin embargo, tales manifestaciones tienen una prevalencia considerable y pueden ser indicativas en el cribado y el aislamiento temprano de los casos de esta enfermedad.

Palabras clave: Coronavirus, Oler, Gusto.

\section{INTRODUÇÃO}

A coronavírus disease 2019 (COVID-19), causada pelo severe acute respiratory syndrome coronavirus 2 (SARS-CoV-2), surgiu no leste da Ásia e rapidamente tornou-se a maior pandemia desde o surto de influenza H1N1 em 1918 (MOEIN ST, et al., 2020). É a terceira zoonose causada por um vírus da família Coronavírus, neste século (LOVATO A e FILIPPIS CD, 2020). Essa infecção tem elevada taxa de transmissão e provocou a morte de milhares de indivíduos em todo o mundo. Febre, dispneia, tosse, artralgia, mialgia, cefaleia, rinorreia e diarreia são os sintomas mais prevalentes dessa enfermidade (LOVATO A e FILIPPIS CD, 2020).

Alterações olfativas e gustativas foram relatadas como um achado clínico raro em pacientes chineses com COVID-19, presentes em somente $5 \%$ dos casos (VAIRA LA, et al., 2020), desse modo inicialmente a anosmia não foi listada pelos autores de Wuhan, China (GILANI S, et. al, 2020). Entretanto, com a disseminação da SARS-CoV-2 na Europa, esses sintomas tornaram-se muito frequentes (VAIRA LA, et al., 2020), chegando a índices de $86 \%$ em sintomas de disfunção olfativa e $88 \%$ em disfunção gustativa em um estudo europeu, índices baseados em relatos dos pacientes (MEINI S, et al., 2020). Tais disfunções estão presentes em, até, $30 \%$ dos pacientes com a infecção pelo SARS-CoV-2 (OBIEFUNA S e DONOHOE D, 2020).

De acordo com a European Rhinology Society, 20 a 60\% de pacientes com COVID-19 tem perda de olfato (LOVATO A e FILIPPIS CD, 2020). Entretanto, essa prevalência pode estar subestimada devido à escassez de estudos que avaliam as funções olfativas e gustativas de forma objetiva e não somente baseados em anamneses e entrevistas (VAIRA LA, et al., 2020). Esses distúrbios podem ocorrer em conjunto com as demais manifestações clínicas ou, isoladamente, nos casos leves no início do período de infecção, antes mesmo do aparecimento de sintomas mais característicos dessa doença (LOVATO A e FILIPPIS CD, 2020; OBIEFUNA S e DONOHOE D, 2020; XYDAKIS MS, et al., 2020).

Desde o início da pandemia, muitos médicos questionaram se a anosmia poderia estar associada a um curso clínico mais leve em pacientes com COVID-19, o que ainda precisa ser mais detalhado (LECHIEN JR, et al., 2020a). Acredita-se que esses sintomas também ocorrem em formas graves, porém nesses casos eles são negligenciados (VAIRA LA, et al., 2020). As causas de disfunções olfativas e gustativas em pacientes com COVID-19 também são pouco conhecidas, sendo sugerido que o epitélio olfatório da cavidade nasal apresente tropismo aumentado para a ligação do SARS-CoV-2, pois as células desse local expressam grande quantidade de dois receptores, a Enzima Conversora de Angiotensina 2 (ECA2) e a serina protease transmembranar 2 (TMPRSS2), que facilitariam a ligação e replicação do vírus neste sítio (BUTOWT $R$ e BILINSKA K, 2020; GIACOMELLI A, et al., 2020). 
No tratamento da anosmia, esteroides orais são desaconselhados nas primeiras 2 semanas na anosmia pós-viral com suspeita de COVID-19 (HOPKINS C, et al., 2020), uma vez que relatos na literatura descrevem o período de 14 dias para normalização da função olfatória (SOLER ZM, et al., 2020). Esses devem apenas ser considerados em casos que essa disfunção persista após 14 dias, considerando riscos e benefícios (HOPKINS C, et al., 2020).

Isto evidencia a importância de uma revisão bibliográfica sobre manifestação de alterações olfativas e gustativas na COVID-19 para reunir e facilitar o acesso às poucas informações sobre esse assunto. Além disso, a taxa de transmissão da COVID-19 é alta, podendo causar disfunções do olfato e paladar, desde suas fases iniciais, antes de outros sintomas mais prevalentes dessa doença ou ocorrerem, isoladamente, nos casos leves. A identificação desses distúrbios, inclusive nos profissionais de saúde, é útil para suspeição, triagem e isolamento precoce dos casos e para se evitar a infecção nosocomial (HOPKINS C, et al., 2020).

\section{REVISÃO BIBLIOGRÁFICA}

\section{Prevalência de anosmia e disgeusia}

A COVID-19, causada pelo SARS-CoV-2 tornou-se uma pandemia e, ao se espalhar pelo continente Europeu, causou uma nova manifestação clínica nos pacientes, a súbita anosmia ou ageusia, além dos sintomas clássicos dessa doença (LECHIEN JR, et al., 2020b). Uma pesquisa, realizada no Reino Unido através de um questionário simples e enviado por email a pacientes que referiam anosmia recente, aos seus amigos e familiares portadores dos mesmos sintomas, obteve um total de 2428 respostas, sendo, $61 \%$ com o começo da anosmia há menos de uma semana, anterior ao preenchimento do questionário, correspondendo ao começo da incidência crescente de COVID-19 no Reino Unido, 16\% não relatou nenhum outro sintoma associado a COVID-19.

Em pacientes que referiram sintomas o mais comum foi tosse, em $43 \%$, rinorreia, em $34 \%$ e febre em $28 \%$. Sobre o início dos sintomas e anosmia foram obtidas 1325 respostas, $13 \%$ referiram anosmia antes dos outros sintomas, $38,4 \%$ ao mesmo tempo e $48,6 \%$ a anosmia veio após os outros sintomas. Dos $2428,74,4 \%$ relataram perda completa de olfato e $17,3 \%$ perda grave. Aproximadamente um a cada quatro indivíduos com anosmia teve apenas esse sintoma ou ele foi o primeiro a se apresentar. Deste estudo, apenas 80 pacientes tinham sido testados para COVID-19 com 74\% de resultados positivos (HOPKINS C, et al., 2020).

A prevalência desses distúrbios é muito variável na literatura, de acordo com a European Rhinology Society e relatórios de pesquisa da Alemanha, Estados Unidos, Grã-Bretanha, Irã, Itália e é de 20-68\% em indivíduos acometidos pela COVID-19 (LOVATO A e FILIPPIS CD, 2020; MOEIN ST, et al., 2020). Esses resultados são semelhantes aos de três estudos. O primeiro, um estudo transversal com 59 pacientes, em um hospital de Milão, Itália, revelou uma prevalência de distúrbios olfativos (anosmia e hiposmia) e gustativos (ageusia e disgeusia), em cerca de $34 \%$ dos pacientes com COVID-19. Desses, cerca de $15 \%$ tiveram desordem do olfato ou do paladar e, aproximadamente, 18,6\% em ambos os sentidos (GIACOMELLI A, et al., 2020).

O segundo estudo acompanhou, no período de 27 de janeiro até 21 de março de 2020, 63 pacientes internados na clínica Schwabing em Munique, Alemanha. Do total, 41\%relataram anosmia e/ou ageusia. Destes, $31 \%$ queixaram-se de rinite concomitante. Além disso, não foram identificadas outras doenças que poderiam afetar o olfato e paladar (BOCKSBERGER, et al., 2020). O terceiro estudo acompanhou a evolução de 59 pacientes positivos para COVID-19 e relatou a ocorrência de distúrbios de olfato e paladar de, respectivamente, $68 \%$ e $71 \%$. Os principais sintomas auto relatados foram: fadiga $(80 \%)$, ageusia $(71 \%)$, febre (70\%), anosmia (68\%), mialgia ou artralgia (63\%), diarreia (48\%) e náusea (27\%) (YAN CH, et al., 2020).

Variações na porcentagem de distúrbios olfativos têm sido relatadas, como em um estudo retrospectivo e observacional de uma série de casos de COVID-19 em um Centro de Tratamento, na China (Union Hospital of Huazhong University of Science and Technology - Wuhan). Dos 214 pacientes em estudo, 5,1\% e 5,6\%, respectivamente, apresentaram comprometimento do olfato e do paladar. Além disso, os sintomas clássicos da doença mais comuns foram: febre $(61,7 \%)$; tosse $(50 \%)$; e anorexia $(31,8 \%)$ (MAO L, et al., 2020$)$. 
A porcentagem destes sintomas foi muito alta entre pacientes com COVID-19 selecionados de 12 hospitais europeus. Neste estudo, $85,6 \%$ dos 417 pacientes analisados na amostra tiveram alteração no olfato pela infecção, sendo anosmia em $79,6 \%$ deles, hiposmia em $20,4 \%$, anosmia em $12,6 \%$ e parosmia em $32,4 \%$. Episódios de rinorreia e congestão nasal estavam associados ao desenvolvimento de alterações olfativas ou gustativas em, apenas, 3,8\% dos pacientes (LECHIEN JR, et al., 2020b). Sobre as alterações na gustação, $88,8 \%$ dos pacientes do estudo tiveram algum distúrbio. A hipogeusia foi encontrada em $78,9 \%$ deles e a distorção do paladar em $21,1 \%$.

No grupo de pacientes sem alteração de paladar, 46,5\% referiam anosmia ou hiposmia. Entretanto, alteração de olfato e gustação concomitantes mostraram significância estatística (LECHIEN JR, et al., 2020b). Investigação acerca das manifestações neurológicas em pacientes hospitalizados com COVID-19 foi realizada na população espanhola contando com uma amostra de 841 pacientes. Destes, 57,4\% desenvolveram sintomas neurológicos, sendo anosmia e disgeusia representados, respectivamente, por 4,9\% e 6,2\% (ROMERO-SÁNCHEZ CM, et al., 2020).

Um estudo de caso-controle realizado com 60 indivíduos positivos para o SARS-CoV-2 e 60 controles, admitidos no Masih Daneshvari Hospital em Teerã, no Irã, demonstrou a diferença entre os déficits olfativos e gustativos percebidos pelo próprio paciente e a existência dos mesmos ao serem testados. $35 \%$ dos pacientes positivos para SARS-CoV-2 afirmaram ter percebido anosmia ou ageusia, com $12 \%$ relatando apenas anosmia, 7\% apenas ageusia e 17\% anosmia e ageusia, porém através do The University of Pennsylvania Smell Identification Test (UPSIT), 98\% dos pacientes apresentaram algum grau de disfunção olfativa.

Desses pacientes, 25\% tinham anosmia, 33\% hiposmia grave, 27\% tinham hiposmia moderada e 13\% hiposmia leve. Essa diferença entre os valores encontrados pelo UPSIT e o relato dos próprios pacientes se deve ao fato de que a maioria desconhece que possui algum déficit olfativo, percebendo-o apenas ao ser testado ou estar anósmico (MOEIN ST, et al., 2020), geralmente em quadros e/ou doenças complicadas acontece essa perda que só é notada pelos pacientes após muito tempo por ser um prejuízo sutil ao paciente (XYDAKIS MS, et al., 2020). Em relação aos controles, nenhum deles relatava ter percebido alterações olfativas e, quando submetidos a testes específicos para detecção, $82 \%$ apresentaram o teste sem alterações e $18 \%$ apenas hiposmia leve (MOEIN ST, et al., 2020).

Outro estudo, de caráter retrospectivo, também realizado em Teerã com pessoas que relataram anosmia teve prevalência de $62,5 \%$ positivos para COVID-19, dos quais $80 \%$ relataram começo da anosmia após 2 a 4 dias de sintomas como febre e dispneia; $25 \%$ apresentaram anosmia, porém não foram testados (GILANI S, et. al, 2020).

Um estudo de coorte, realizado em Paris, avaliou os sintomas clínicos de 1487 pacientes com COVID-19. Dentre os sintomas mais prevalentes encontram-se a anosmia, relatada por $28 \%$ dos pacientes, a ageusia (28\%) e a ageusia e anosmia concomitantes (23\%) (LAPOSTOLLE F, et al., 2020). O sintoma mais comum relacionado aos distúrbios olfativos é anosmia, mas outro trabalho também verificou uma alta porcentagem de sintomatologia olfatória e gustativa na Espanha, com 80,6\% de 79 pacientes com COVID-19 tendo disfunções olfativas, cuja manifestação mais comum foi a anosmia $(42,2 \%)$.

Além disso, 90,3\% dos pacientes relataram disfunções no paladar, sendo ageusia o sintoma mais comum (45,2\%) (BELTRÁN-CORBELLINI Á, et al., 2020). Isto reforça dados de centros especializados em olfato e paladar, que afirmam ser a hiposmia o sintoma mais comum, podendo atingir dois terços dos pacientes com disfunções olfativas, devido a todas as etiologias, seguida por anosmia (SOLER ZM, et al., 2020).

A prevalência das alterações do olfato tem sido maior em indivíduos mais jovens e em pessoas do sexo feminino (BELTRÁN-CORBELLINI Á, et al., 2020; GIACOMELLI A, et al., 2020; LECHIEN JR, et al., 2020b). Uma possível explicação desta última associação seria pela natureza da reação inflamatória, que classicamente difere entre os gêneros (LECHIEN JR, et al., 2020b). 
Além disso, os distúrbios agudos de olfato e paladar podem ser sinais clínicos para suspeição, triagem e isolamento precoce dos casos com um controle mais eficiente da pandemia da COVID-19 (BELTRÁNCORBELLINI Á, et al., 2020). Isto está de acordo com o proposto em 22 de março de 2020 pela American Academy of Otolaryngology - Head and Neck Surgery, de que a anosmia e disgeusia deveriam ser adicionadas como sintomas na lista de screening para casos suspeitos dessa doença (LOVATO A e FILIPPIS CD, 2020).

\section{Surgimento dos sintomas}

Distúrbios olfativos surgiram, previamente, ou, simultaneamente, aos outros sintomas (BELTRÁNCORBELLINI Á, et al., 2020; GIACOMELLI A, et al., 2020; YAN CH, et al., 2020). 60\% dos pacientes com desordens do paladar ou olfato identificado foram sintomáticos antes da internação e $40 \%$ desenvolveram os distúrbios durante a internação. Além disso, $22 \%$ e $35,5 \%$ dos pacientes, em dois estudos, relataram anosmia como primeiro sinal clínico (BELTRÁN-CORBELLINI Á, et al., 2020; YAN CH, et al., 2020). Em outra casuística, cerca de $60 \%$ dos casos apresentaram tais sintomas desde início da doença, sendo 3,5 dias o tempo médio para manifestação (ROMERO-SÁNCHEZ CM, et al., 2020). Isto concorda com outros relatos de que tais desordens são comuns em pacientes infectados pelo SARS-CoV-2 (GIACOMELLI A, et al., 2020) e que podem ocorrer, em conjunto com os demais sintomas clínicos, ou, isoladamente nos casos leves ou logo no início do período de infecção (OBIEFUNA S e DONOHOE D, 2020).

A relação entre a infecção pelo SARS-CoV-2 e os distúrbios recentes de olfato e paladar foram citados na maioria dos estudos. Por isto, esses sinais clínicos poderiam ser usados para teste e controle precoce de casos suspeitos os quais seriam submetidos a tratamento e/ou quarentena (BELTRÁN-CORBELLINI Á, et al., 2020; MOEIN ST, et al., 2020), uma vez que podem representar estágios iniciais da infecção (GIACOMELLI A, et al., 2020).

\section{Fisiopatologia}

As causas de disfunções olfativas e gustativas em pacientes com COVID-19 são pouco conhecidas. Teorias para explicar as disfunções de olfato e paladar, em função dessa doença, incluem serem devidas a coriza, a obstrução nasal e a faringite pela relação da resposta inflamatória e do edema com o prejuízo na função de olfato e paladar. Essas ocorrências não estavam associadas em muitos pacientes e perdas sensoriais, inclusive, precederam tais sintomas. O SARS-CoV-2, como qualquer infecção, está implicado no estímulo à produção de anticorpos pelo hospedeiro, os quais, possivelmente, podem causar danos às células dos sistemas olfativo e gustativo. Muitos pacientes com COVID-19 recebem tratamento com antibióticos, antitérmicos ou antivirais e esses medicamentos podem ocasionar alterações transitórias de olfato e paladar. No entanto, muitos doentes desenvolvem tais alterações sem uso de medicações (FINSTERER $\mathrm{J}$ e STOLLBERGER C, 2020).

Sintomas neurológicos da COVID-19 (MAO L, et al., 2020; PARRA JED, et al., 2020) podem ser divididos em: manifestações no Sistema Nervoso Central, Sistema Nervoso Periférico e em injúrias na musculatura esquelética. $\mathrm{O}$ acometimento neurológico, quando ocorre, está presente, na maioria das vezes, no início da doença (MAO L, et al., 2020; ROMERO-SÁNCHEZ CM, et al., 2020). Isto corrobora a hipótese de que as disfunções no olfato podem ser causadas por alterações de fundo neurológico, pois os sintomas olfatórios acontecem - ou se iniciam - em sua maioria, antes de outras manifestações características da COVID-19, como febre e tosse (LOVATO A e FILIPPIS CD, 2020).

Disfunção olfativa inicial, no quadro dessa infecção, quando nenhum outro sintoma está aparente, seria devido à infecção ter atingido o sistema nervoso periférico, especificamente o neuroepitélio, levando a sintomatologia. Por outro lado, a rápida recuperação das disfunções olfativa e gustativa (MEINI S,et al., 2020), testemunha contra essa hipótese. Isso porque, em outras infecções virais que também apresentam disfunção olfativa e cuja causa conhecida disso é a infecção do neuroepitélio, dos pacientes que apresentam a sintomatologia após resolução do quadro infeccioso, $80 \%$ deles relatam melhora do quadro apenas um ano ou mais depois da resolução da doença (MEINI et al., 2020). 
O mecanismo fisiopatológico da instalação da anosmia não é bem conhecido. As principais teorias para explicar o mesmo são uma resposta imunológica rápida mediada por citocinas; lesão direta dos neurônios olfatórios e expressão no epitélio nasal das proteases ligadas a instalação e replicação do vírus, devido a altos níveis de ECA2 e TMPRSS2 no local (BUTOWT R e BILINSKA K, 2020; LOVATO A e FILIPPIS CD, 2020). No momento, uma das grandes questões a ser esclarecida é se essas proteases são expressas em células neuronais ou não neuronais da mucosa nasal, ou se estão presentes em ambas. A presença nas células neuronais, podem levar esses receptores a um transporte axonal anterógrado do vírus pelo nervo olfatório até o sistema nervoso central. Porém, se as proteases ECA2 e TMPRSS2 encontrarem-se, apenas, nas células não neuronais da mucosa nasal, isso estabilizaria a carga viral, apenas, neste sítio, sem sua disseminação local por essa via (BUTOWT R e BILINSKA K, 2020).

A família Coronavírus é classificada como sendo composta por vírus respiratórios, porém, são conhecidos por serem neuroinvasivos e neurotrópicos, podendo a via respiratória comportar-se como uma porta de entrada (XYDAKIS MS, et al., 2020). O epitélio olfatório da cavidade nasal mostra-se como um local provável de tropismo aumentado para a ligação do SARS-CoV-2, em função das células desse local expressar em grande quantidade dois receptores, as proteases ECA2 e TMPRSS2, que facilitariam a ligação e replicação do vírus neste sítio (BUTOWT R e BILINSKA K, 2020). De igual forma a protease ECA2 é altamente expressa no epitélio da mucosa oral (GIACOMELLI A, et al., 2020).

O efeito citopático viral nas células do epitélio olfativo pode alterar a neurotransmissão, e o receptor ECA2 tem papel na regulação da enzima DOPA-descarboxilase, desse modo a infecção pelo SARS-CoV-2 pode estar relacionada a uma menor produção de dopamina e serotonina (FINSTERER J e STOLLBERGER C, 2020). Além disso, os vírus SARS-CoV-2 mostraram capacidade de infectar diversos locais do corpo (FINSTERER J e STOLLBERGER C, 2020), inclusive o sistema nervoso, pois os ácidos nucleicos de SARSCoV-2 foram identificados no líquor e no tecido cerebral em autópsias de pacientes acometidos pela COVID19 (MAO L, et al., 2020).

O tratamento de alterações do olfato teve $16,7 \%, 8,1 \%, 2,5 \%$ e 2,5\% dos pacientes tratados com solução salina nasal, corticoides nasais, corticoides orais e outras opções de terapêuticas, respectivamente. A Sociedade Francesa de Otorrinolaringologia não recomenda o uso de corticoides em indivíduos com COVID19 (LECHIEN JR, et al., 2020b) e esteróides orais são desaconselhados nas primeiras duas semanas, para o tratamento da anosmia pós-viral em pacientes com suspeita de COVID-19 (HOPKINS C, et al., 2020), pois a normalização da função olfatória se dá em período de 14 dias (SOLER ZM, et al., 2020). Não existe comprovação de benefício com o uso destes medicamentos neste contexto, somando-se a isso a preocupação com possíveis malefícios do uso de corticoterapia em pacientes infectados (SOLER ZM, et al., 2020).

Esses compostos devem ser considerados, apenas, quando essa disfunção persistir por mais 14 dias (HOPKINS C, et al., 2020) ou nos casos de perda olfativa relacionada ao efeito citopático viral, ou seja, pacientes que permanecem sintomáticos após a resolução da infecção (SOLER ZM, et al., 2020). A possibilidade de uso nestes casos destas medicações por um breve período não foi, ainda, completamente comprovada (HOPKINS C, et al., 2020; SOLER ZM, et al., 2020).

O treinamento olfativo, que oferece estímulos odorantes ao sistema olfatório e contribui para a regeneração do mesmo pode ser, alternativamente, empregado com duas sessões diárias nas quais o paciente inspira por 15 segundos quatro odores diferentes, perfazendo uma duração mínima de seis meses de terapia. Entretanto, os resultados desta terapia têm sido, na maioria das vezes, modestos e os pacientes idealmente devem ser acompanhados por um otorrinolaringologista (SOLER ZM, et al., 2020).

\section{Melhora Clínica}

A resolução das alterações do olfato variou de 10\% (YAN CH, et al., 2020) a 63\% (LECHIEN JR, et al., 2020b) dos pacientes com quadro clínico clássico da doença (YAN CH, et al., 2020). O processo de cura mostrou que o tempo de recuperação, dos pacientes, variou de um a oito dias após a resolução dos sintomas clássicos (LECHIEN JR, et al., 2020b) com média de 7,4 $\pm 2,3$ dias (BELTRÁN-CORBELLINI Á, et al., 2020). 
A melhora dos sintomas olfativos após seu início ocorreu entre uma a quatro semanas, com $72,5 \%$ dos pacientes referindo melhora espontânea (YAN CH, et al., 2020). A perda da função olfativa pode ocorrer, também, em pacientes em processo de recuperação da COVID-19, pois aqueles avaliados pelo UPSIT iriam receber alta do serviço médico em até quatro dias (MOEIN ST, et al., 2020).

Um estudo feito em Florença, na Itália, teve como objetivo analisar o tempo de recuperação das disfunções olfativas e gustativas em pacientes hospitalizados, devido a COVID-19, em nível não intensivo. Por meio de contato telefônico um mês após a alta do paciente, dos 100 pacientes incluídos no estudo, 42 deles relataram algum grau dessas disfunções em algum momento da doença, predominando em homens (razão 2:1). 0 tempo de recuperação médio foi de 18 dias para a disfunção olfativa e de 16 dias na gustativa, com as mulheres apresentando um tempo de recuperação médio maior (26 dias nas mulheres versus 14 dias nos homens). Em um mês de alta, dos 42 pacientes que apresentaram esses sintomas, 64\% relataram melhora completa e $19 \%$ relataram melhora quase completa, totalizando $83 \%$ dos pacientes com a disfunção resolvida no acompanhamento após um mês (MEINI S, et al., 2020).

\section{CONSIDERAÇÕES FINAIS}

Esta revisão permitiu mostrar a escassez de trabalhos relacionando disfunções olfativas e gustativas com a COVID-19 e de informações consolidadas sobre esse tema. Isso se deve ao fato de a doença ser recente e muitos estudos ainda estarem em andamento. Além disso, essas manifestações clínicas tem prevalência considerável e podem ser os únicos ou primeiros sintomas a surgirem auxiliando a identificação, suspeita diagnóstica, triagem e isolamento precoce dos casos, promovendo um controle mais eficiente da pandemia.

\section{AGRADECIMENTOS}

Ao Dr. Thales Pardini Fagundes, pelo incentivo e atenção no esclarecimento de pontos importantes para a redação deste artigo. E ao professor PhD. José Cola Zanuncio, pela revisão e melhorias no texto.

\section{REFERÊNCIAS}

1. BELTRÁN-CORBELLINI Á, et al. Acute-onset smell and taste disorders in the context of Covid-19: a pilot multicenter PCR-based case-control study. European Journal of Neurology, 2020; 0: 1-4.

2. BOCKSBERGER S, et al.Temporary hyposmia in COVID-19 patients. HNO, 2020; 68(6): 440-443.

3. BUTOWT R, BILINSKA K. SARS-CoV-2: Olfaction, Brain Infection, and the Urgent Need for Clinical Samples Allowing Earlier Virus Detection. ACS Chemical Neuroscience, 2020; 11(9): 1200-1203.

4. FINSTERER J, STOLLBERGER C. Causes of hypogeusia/hyposmia in SARS-CoV2 infected patients. Journal of Medical Virology, 2020; 0: 1-2.

5. GIACOMELLI A, et al. Self-reported olfactory and taste disorders in SARS-CoV-2 patients: a cross-sectional study. Clinical Infectious Diseases 2020; DOI: 10.1093/cid/ciaa330.

6. GILANI S, et al. COVID-19 and Anosmia in Tehran, Iran. Medical Hypotheses, 2020; 141:109757.

7. HOPKINS C, et al. Presentation of new onset anosmia during the COVID-19 pandemic. Rhinology, 2020; 58(3):295298.

8. LAPOSTOLLE F, et al. Clinical features of 1487 COVID-19 patients with outpatient management in the Greater Paris: the COVID-call study. Internal and emergency medicine, 2020; DOI: 10.1007/s11739-020-02379-z.

9. LECHIEN JR, et al. Objective olfactory evaluation of self-reported loss of smell in a case series of 86 COVID-19 patients. Head and Neck, 2020; 42:1583-1590.

10. LECHIEN JR, et al. Olfactory and gustatory dysfunctions as a clinical presentation of mild-to-moderate forms of the coronavirus disease (COVID-19): a multicenter European study. European Archives of Oto-Rhino-Laryngology, 2020; 277(8):2251-2261.

11. LOVATO A, FILIPPIS CD. Clinical Presentation of COVID-19: A Systematic Review Focusing on Upper Airway Symptoms Ear, Nose and Throat Journal, 2020; DOI: 10.1177/0145561320920762.

12. MAO L, et al. Neurologic Manifestations of Hospitalized Patients with Coronavirus Disease 2019 in Wuhan, China. JAMA Neurology, 2020; 77(6):683-690.

13. MEINI S, et al. Olfactory and gustatory dysfunctions in 100 patients hospitalized for COVID-19: sex differences and recovery time in real-life. European archives of oto-rhino-laryngology, 2020; DOI: 10.1007/s00405-020-06102-8.

14. MOEIN ST, et al. Smell dysfunction: a biomarker for COVID-19. International Forum of Allergy \& Rhinology, 2020; DOI: $10.1002 /$ alr.22587. 
15. OBIEFUNA S, DONOHOE C. Neuroanatomy, Nucleus Gustatory. 1st ed. Treasure Island (FL): StatPearls Publishing, 2020; 7p.

16. PARRA JED, et al. EI COVID-19 también Afecta el Sistema Nervioso por una de sus Compuertas: El Órgano Vascular de la Lámina Terminal y el Nervio Olfatorio. Alerta Neurológica, Prueba de Disosmia o Anosmia Puede Ayudar a Un Diagnóstico Rápido TT - COVID-19 also Affects the. International journal of odontostomatology, 2020; 14 (3): 285 287.

17. ROMERO-SÁNCHEZ CM, et al. Neurologic manifestations in hospitalized patients with COVID-19: The ALBACOVID registry. Neurology, 2020; DOI:10.1212/WNL.0000000000009937.

18. SOLER ZM, et al. A primer on viral-associated olfactory loss in the era of COVID-19. International forum of allergy \& rhinology, 2020; DOI: 10.1002/alr.22578.

19. VAIRA LA, et al. Olfactory and gustatory function impairment in COVID-19 patients: Italian objective multicenterstudy. Head \& neck, 2020; 42:1560-1569.

20. XYDAKIS, MS, et al. Smell and taste dysfunction in patients with COVID-19. The Lancet Infectious Diseases, 2020; DOI: 10.1016/S1473-3099(20)30293-0.

21. YAN CH, et al. Association of chemosensory dysfunction and Covid-19 in patients presenting with influenza-like symptoms. International forum of allergy \& rhinology, 2020; 10: 806- 813. 